\title{
A GSM MODULE-BASED SMART ELECTRIC METER READER
}

\author{
Opeyemi OSANAIYE*, Sunday UNOGWU**, Folayo AINA ${ }^{* * *}$ \\ "Nile University of Nigeria, Department of Computer Engineering, Abuja Nigeria, tel: +2348059879274 , \\ E-mail: opeyemi.osanaiye@nileuniversity.edu.ng \\ ${ }^{* *}$ Federal University of Technology, Minna Niger State, Nigeria, \\ E-mail: sunday.unogwu@st.futminna.edu.ng \\ ${ }^{* * *}$ School of Psychology and Computer Science, University of Central Lancashire, United Kingdom, \\ E-mail: faina@uclan.ac.uk
}

\begin{abstract}
The traditional and estimated billing system of electric energy consumed in most part of Sub-Saharan Africa has become a lingering issue to the electricity consumers. This has therefore necessitated the advent of smart electric meters. In this work, we propose a smart electric meter reader that provides an efficient and economically viable technique for measuring the consumption of electricity. This proposed method tends to solve many issues of the traditional reading system, such as reading efficiency, accuracy, and the elimination of human interface. Our proposed method, consisting of a GSM module, is used to wirelessly communicate the smart meter readings to the electricity provider and the consumer in form of a text message. The results obtained from the evaluation of this work show that our proposed method has improved the accuracy of the meter reading process for proper accountability.
\end{abstract}

Keywords: IoT, Smart Meter, Electric Meter, GSM Module

\section{INTRODUCTION}

Electricity is very essential in every country, as it is one of the key factors in the development of any nation, hence the rate at which it is consumed is ever increasing. The rate at which industrial, commercial and residential consumers of electricity are increasing across the globe has necessitated service providers to devise a better, efficient and environmentally safe technique of measuring electricity consumption. This ensures that accurate bills are generated and invoiced. Electrical metering device technology has come a long way from what it used to be many years back. From the old bulky electricity meters with huge magnets and coils, there are currently ongoing innovations to reduce the size and weight in addition to improving its features and specifications. Electric energy meter is an instrument (device) that measures the quantity of electricity consumed by consumers over a specified period of time in residential, commercial and industrial buildings. Electric energy meters are usually calibrated in billing units, often a times in kilowatt-hour.

Accuracy and resolution of the electric meter have experienced significant improvements over the years. The development and deployment of digital electrical meters in the later part of last century has totally changed the way electricity consumption is being measured. Starting with the ammeters and voltmeters, the digital electrical meters have subdued most of the measuring instruments of the past, due to their advantages, which include ease in taking readings, rugged construction and enhanced resolution. Notable is the introduction of the electronic meters in the mid-eighties [1]. Traditionally, in Nigeria, the electricity company, Nigeria Electric Power Authority (NEPA) billing started manually using analog meter [2]. The analog electric meter measures only the quantity of electricity supplied to the consumer. The most prevalent types of the analog electric meters are often called kilowatt-hour meters. The kilowatt-hour is a very convenient unit for electricity billing, because the electricity utility usage of an average consumer of electricity in a month is several hundred of kilowatt hours. Higher units like megawatt- hours and terawatt-hours are used for metering of huge amount of electrical energy [3]. The manual process of gauging utility involves meter reader personnel visiting premises of the consumer to take the record of the readings before bill can be generated and invoiced. Manual system that is employed is cumbersome and quite defective. It only makes the process more difficult and complex. Technological advancement, coupled with the rise in the number of electricity consumers, has led to the computerization of the billing system [4]. This has necessitated the production of prepaid electricity billing meter. Different from the usual analogue electric meter that is used for generating the billing charges, the Smart Electric Meter Reader (SEMR) is a device that is able to automatically send periodical readings of the amount of electricity consumed to a server in the billing room of the electricity supply company and the consumer of the electricity wirelessly.

Traditionally, electric meters are usually situated at the consumer's vicinity and the information (i.e. the meter readings) of the consumption of electricity is collected by the electricity utility workers, usually called meter readers, on their monthly or fortnightly visits to the premises. This method of gauging utility consumption of electricity has several disadvantages. Among which are; hindered access to the location of the meter, cost of hiring meter-readers and invasion of customer's privacy.

\section{BACKGROUND}

\subsection{Traditional Electric Meter}

Historically, the invention of dynamo in the 1860s made generation of large amount of electricity possible. Lighting was the foremost mass commercial application of this electrical energy. At the beginning of selling this new electrical energy product, it was obvious that the cost needs to be determined. The need to measure the amount of electricity to determine the cost of consumption necessitated the invention of energy meter (electric meter). The increase in the commercial use of electricity in the 
1880s made electric meter increasingly important, as the meter was needed to bill consumer properly. The invention of the energy meter made it possible for electricity companies to measure the amount of electrical power a customer had used.

Thomas Edison in 1881 developed the first method of measuring the amount of power consumed. He used the method of electrochemical effect of current to measure ampere per hour [5]. Edison installed a jar containing two plate of zinc immerged in an electrolyte of zinc-sulfate. When electricity begins to flow through the jar, the zinc begins to dissolve off the positive plate and onto the negative plate. The workers check every month to see how much of the zinc had moved from the positive plate onto the negative plate. Another meter developed in the 1880 s were the electromechanical meters that dominated the measurement of electrical energy before 1970. Only the measurement of electrical energy was possible with the electromechanical meters. Over time, the requirements of a meter to measure the electrical energy and communicate it, together with other electricity parameters was identified. Hence, the introduction of the solid-state electronic meter that could measure the overall electrical energy parameters [6].

Between 1970 and 2000, electronic meters had been equipped with an additional automatic reading functionality that could send data in near real time. Automatic meter reading system is a technology used in collecting data from energy metering devices before been sent to a central station in order to further process it for the purpose of billing. The automatic meter reading system helps the energy service provider to access the accurate and updated data from the meters. This system can measure energy consumption on hourly, monthly or yearly bases on the request of the utility company. However, it could only provide a one-way communication. In recent years, there has been a shift to a more advanced technology called the smart meter, which eliminates the limitation of the automatic meter reading.

A Smart meter [7] is an electronic device which records the consumption of electricity within a time interval and communicates the measured information to the supply company of the electricity. It provides a two-way communication. Smart meters, like electronic meters, can gauge all the electric power parameters and transmit the data in a way that is meaningful. The consumer of electricity is updated with the amount of electricity consumed, tariffs, cost and other necessary information sent by the provider of the electricity utility. Smart meter has various functionalities to monitor and operate the enduser loads and runs them in an optional means to reduce the electricity bill and also conserves the electrical energy.

Smart meters have been developed over decades ago. However, many developers and researchers are trying to implement new functionalities to the smart meters in other to provide the best solutions for energy conservation, cost effectiveness and demand management. The smart meter technology is constantly changing and many technology institutions and government bodies around the globe are working on how to standardize them [6]. It has variety of technologies and design, but they all operate through a simple process. Smart meters gather measured electricity data and transmits it remotely or locally through a Wide
Area Network (i.e. WAN) or Local Area Network (i.e. LAN) respectively to information collector station [7]. According to information need and use, this data transmiss ion can occur as often as 20 minutes and occasionally as $\mathrm{d}$ aily or weekly. The collector receives the measured data and may or may not perform any further processing of the received data. The data is transmitted through a Wide Area Network (i.e. WAN Network) to the central collection point of the utility for processing, business applications and other use [8]. Since it is a two-way communications path, commands or signals can be sent directly to the meter, distribution device or customer premises.

Smart meter system can be categorized into two, based on the communication technology employed, as defined by their LAN. They are; Power Line Carrier (PLC) and Radio Frequency (RF) [9]. Both categories of the smart meter technology have their own advantages and disadvantages in design and application. The electricity utility company chooses the most appropriate technology that suits its business and demographic needs. Some of the factors that influence the selection of the technology employed include existing infrastructure evaluation, functionality, impact on legacy equipment, technical requirements and the economic impact on the customers of the electricity utility. Selecting the technology to be used involves a detailed analysis and assessment of future requirements and current needs into a single extensive business matter [8].

Power line carrier technology has benefits that include maximizing the use of existing power grid facilities, such as poles and cables, more effective in challenging terrain, improved cost efficacy for rural lines and allowing long distance coverage. Some of the main disadvantages of power line carrier include high latency (it takes longer time to transmit data), less throughput, lower bandwidth, interface with distribution automation devices is limited and high cost in suburban and urban centres. Radio frequency on the other hand are of two types, the mesh technology and the point-to-point technology.

In mesh technology, the smart meters talk to each other (hop) to form a local area network, LAN cloud. The collector transmits information to a central location of the power provider using various wide area network approaches. Mesh Radio Frequency (RF) system benefits include broad bandwidth, acceptable latency, and typically operate at $9157 \mathrm{MHz}$ frequencies. Mesh RF systems also has some shortcoming which include distance and terrain issues for rural areas, several collection centres and proprietary communications. In point-to-point technology, the smart meters communicate directly with the collector, which is mostly a tower. The tower collector transfers the information using different means to the electricity utility central point for further processing. Point-to-point RF systems have some benefits which include little or no delay, broad bandwidth for better performance (better throughput), direct communication with each endpoint, most are spectrum approved, and can travel longer range of distance. Some of the shortcoming of point-to-point Radio Frequency system include licensing $(900 \mathrm{MHz}$ is not licensed), patent communications used for some technologies, terrain in rural areas may prove challenging (i.e. problem of Line of Sight) and interface with distribution automation devices is limited. 


\subsection{Related Works}

Researchers, over the years, have proposed different methods to achieve an accurate measurement of electricity consumed. Barone et al. [10] proposed a combination of both smart metering and charging to help local energy communities. The smart meter is deployed in residence with communication between the smart meter and the aggregator through a cloud database. The smart meter function by sampling the voltage and current hundred times every $20 \mathrm{~ms}$ with a default frequency of $5 \mathrm{KHz}$ which can be increased if necessary. The average values are locally saved on a memory card and sent to the cloud database on Amazon Web Service Platform. The smart meter is mounted on an ESP8266 chip to connect to the local WiFi. $\mathrm{Wu}$ et al. [11] in their work proposed an energy metering chip composed of a flexible computing engine made up of a metering program and a dedicated DSP. The chip can be deployed in general energy metering, direct current energy metering, fundamental energy metering and other forms of measuring and metering of electric signals.

Burunkaya, et al. [12], developed an automatic wireless meter reading system using Zig-Bee wireless communication technology. Zig-Bee wireless communication technology is a series of communication protocols that is used to implement small personal networks using electronic low-power radios that is based on the standard IEEE 802.15.4. Due to the low cost of this wireless communication system, Zig-Bee is often used in systems of remote surveillance and control. Zig-Bee has been designed to deliver high data performance in applications where the duty cycle is not high, and consumption of low power is an important consideration. One major demerit of this work is that a typical Zig-Bee-based device's distance coverage is limited to 10-100 meters, however, this can be expanded further using Zig-Bee system mesh networks.

A PLC modem was developed for automatic meter reading in [13]. This system measures and analyses the energy used by customers in a locality and transmits the measured readings to the customer and the electricity provider using power line communication system (PLC) [13]. Power Line Communication (PLC) is a communication system that allows metering data to be transmitted over existing power cables. It serves as an effective device for measuring (metering), controlling and communicating customer's power consumption to the utility company via the power line and regulating loads from a centralized control tower. The main advantage of using PLC is that it does not require extra wires, but interference and noise makes it ineffective.

An automatic meter reading system that uses an open source hardware and software has been proposed in [14]. In the design of this system, the AMR uses Wi-Fi communication protocol to transmit data to a remote server [14]. The data is sent within a specified period with the information packet containing all that the AMR device user needs to know. The duration of the data transfer is defined in the hardware where the service provider sets the parameters [14]. Transferring packages through Wi-Fi protocols is within the HTML (Hyper-Text Markup Language) 1.0 framework, which is also a method for displaying selected content (data) on the device selected.
The user of the system is able to access the displayed data on any device that has the capability to connect to Wifi communication bus bar (mobile devices such as phone tablets, portable personal computer, etc.), and recognizes the HTML 1.0 scripting language. Similarly, Wen-xin Lei et al. [15] have proposed a new ARM system that adds the ability of edge computing on smart grid to achieve a drop in power consumption from the main station of the power grid system to the edge of the network. This achieves new functions like real-time services and efficient data processing

Viciana et al. [16] proposed OpenZmeter (oZm), an open source, low cost, precise and reliable power and electric energy meter that can be installed in houses in both rural and urban areas. oZm has an analog front end that captures the current and voltage waveforms. The oZm get its energy from the grid by using an isolated $\mathrm{AC} / \mathrm{DC}$ source which provides energy for the circuitry. Alternatively, a lithium-ion battery is used to power the board to keep the system running for hours during cases of power cut or low voltage. Mustafa et al. [17] proposed a protocol that uses Multiparty Computations (MPC) as the cryptographic primitive to securely and privately aggregate metering data used for operational purposes such as calculating the power generated, transmitted and billing fees. To secure transmitted data in smart metering system, Kumar et al. [18] propose a lightweight authentication and key management (LAKA) that ensures integrity, anonymity, trust and adequate security in the smart energy network using hybrid cryptography. LAKA achieves two-way authentication between a remote smart meter and the neighbourhood area network and obtains session key agreement to secure data communications.

In this work, we propose a GSM Module-based Smart Electric Meter Reader, herein referred to as GMSEMR, that reads and transmit consumed energy to both the consumer and the database of the utility company for billing and other analysis. Our proposed system consists of three main elements, the smart wireless electric meter reader, the utility company database and the consumer's mobile phone. The GSM Module-based Smart Electric Meter Reader (GMSEMR) will be installed in the consumers premises where it will be connected to electrical power outlet to read the amount of energy consumed. The system enables the service providers and customers to access an updated and accurate record of the readings from the meter. It sends periodical reading of the electric meter wirelessly to a server database in the billing room of the electricity utility company and to the consumer mobile device. The device enables a two-way reading; one on an LCD display and the other as SMS. The introduction of the GSM module ensures the data is transmitted wirelessly and not distance bound.

The main contribution of this work is the introduction of both voltage and current sensor that accurately measures the consumed power and transmits to both the LCD display and the consumer though SMS, using a GSM module.

The rest of the paper is organised as follows. Section 3 describes the proposed system design while Section 4 presents the results and analysis. Section 5 concludes the work. 


\section{SYSTEM DESIGN}

In designing GMSEMR, software and hardware component are required. The software component is a programming language that gives instruction to the device.
The hardware component consists of several parts such as the power supply unit, the control unit, the sensing unit and transmitting unit which are all integrated to form the entire system. The block diagram of the entire system is shown in Fig. 1.

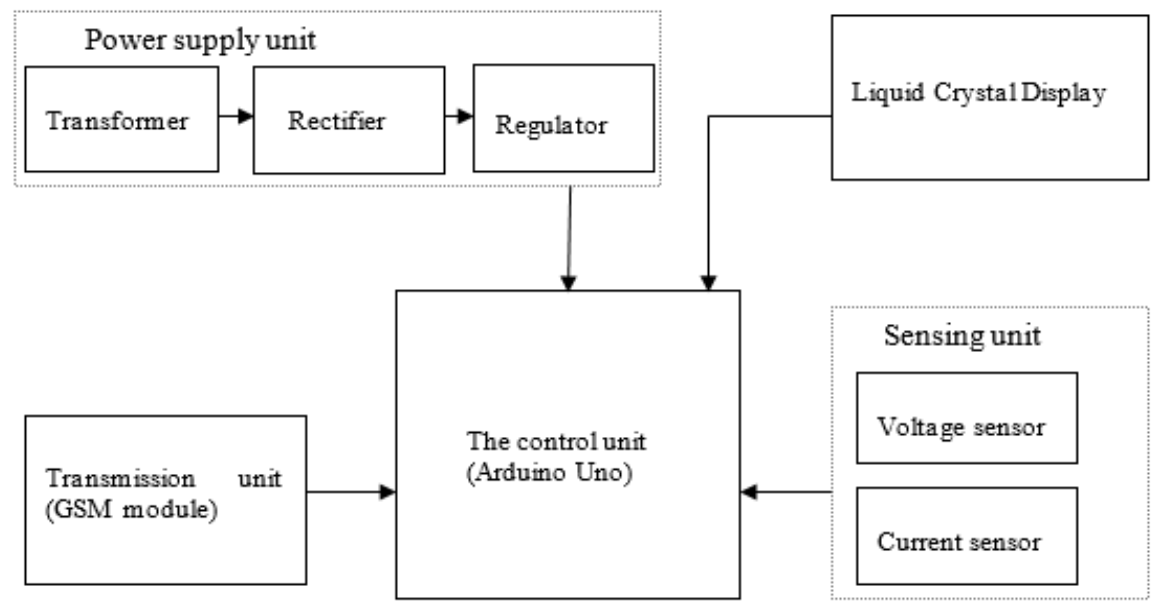

Fig. 1 Block diagram of the wireless electric meter

For the software design, we deploy GMSEMR Arduino IDE application software (See Fig. 2). This application software is used for writing and debugging programs (set of instructions) which is uploaded to the Arduino microcontroller. It has a text editor, debuggers and several libraries that help in the writing the program.

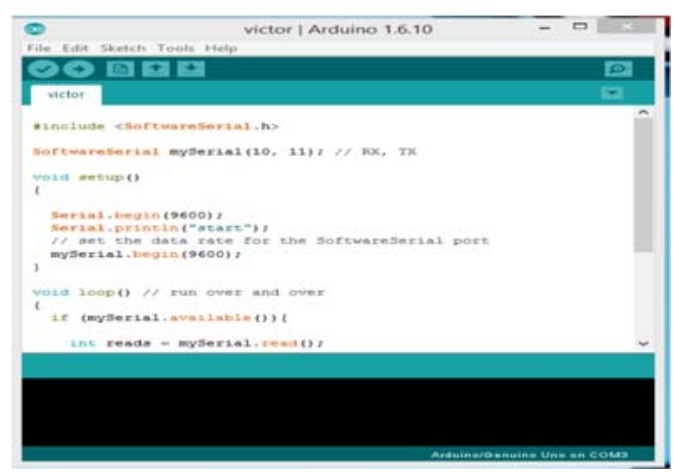

Fig. 2 The Arduino IDE

For the hardware design, components such as, power supply unit consisting of transformer, bridge rectifier, filter and voltage regulator; Arduino Uno (Microcontroller); Voltage Sensor; Current Sensor; Liquid Crystal Display (LCD) and GSM module were used.

The transformer in the power supply unit is the device used to increase or decrease the free flow of an alternating current. A step-down transformer was used in the design to step down the 220-volt AC from the mains to 12 -volt AC. The voltage of the primary and secondary windings are directly proportional to the number of turns of the windings but inversely proportional to the current of both windings. The equation expressing this relation is given as: $\mathrm{E} 1 / \mathrm{E} 2=$ $\mathrm{N} 1 / \mathrm{N} 2=\mathrm{I} 2 / \mathrm{I} 1$, where $\mathrm{E} 1$ and E2 are the voltage of the primary and secondary winding, $\mathrm{N} 1$ and N2 are the number of turns of the primary and secondary winding. I1 and I2 are the current in the primary and secondary windings. Rectification in the design of GMSEMR is necessary as most of the electronic component of the device is DC operated. The rectifier is used to convert an alternating current to direct current, which is the default current of most electronic device. A bridge rectifier is used for this design. The advantage of the bridge rectifier is that it enables both the positive and negative half cycle to be rectified. The Filter used in the design smoothens signals or waveform by removing ripples from the original signal. This is achieved through the use of an electrolytic capacitor. This capacitor can also be used for delay circuit, where safety of equipment from voltage spikes needs to be maintained. The voltage regulator used in the PSU enables the 12-volt dc obtained from the rectifier to be regulated to a specific voltage. In this work, LM 7805 was used to regulate 12 volts to 5 volts.

The Arduino Uno is a microcontroller which is based on atmega 328. Embedded in the board is a microchip where programs are stored. The Arduino program are written on the Arduino IDE and uploaded to it. In this design, the analog pins of the Arduino are connected to the analog sensor. Information are fed into the arduino through its inputs, which are the sensors. When it receives this information, it responds accordingly (as programmed), thereby producing an output. The Arduino Uno has 12 digital pins and 5 analog pins. The schematic of the Arduino Uno board is shown in Fig. 3.

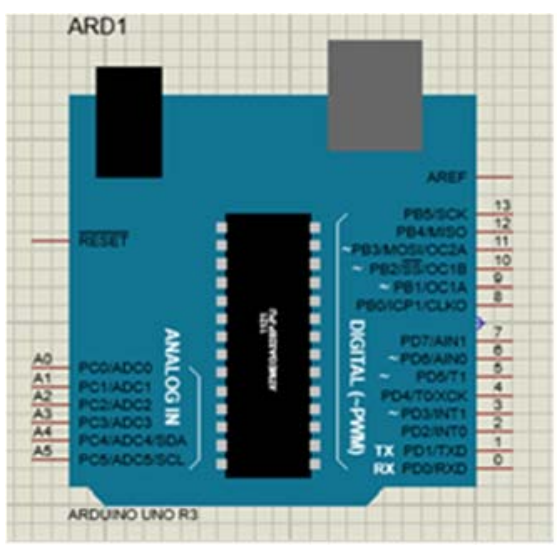

Fig. 3 Schematic of the Arduino Uno board 
The voltage sensor consists of a voltage divider network. The voltage sensor is capable of measuring voltage from 0 to 25 volt. It has four terminals which are the Vcc, Gnd, sense and - terminals. As earlier stated, the voltage divider consists of two resistor of value $35 \mathrm{~K}$ ohm and $7.5 \mathrm{~K}$ ohm which gives a 5:1 voltage divider. The Vcc pin and the Gnd pin must be connected to an external source voltage which is the voltage required to be measured while the sense terminal is the analog input which is connected to the analog input of the microcontroller. The - terminal is connected to the ground pin of the circuit.

For the current sensor, in the design, the principle of ohm's law was used to estimate the current flowing through a small shunt resistor which is connected in series with the voltage sensor. A choke resistor of value 0.22 ohms was used and by applying ohm's law, the current across it will be estimated. The current obtained is very small, therefore, necessitating an operational amplifier used to amplifier the current.

The liquid crystal display is a module that enables text to be displayed. The text shows the status of the input. A 16 by 2 LCD is used in this project which has 16 columns and 2 rows. It is operated by a 5 volt dc supply which is obtained from the 7805-voltage regulator.

A GSM module is a circuit or chip that is used to establish communication using wireless communication technology. The GSM module enables the transmission of data to the receiver. The sim 900 GSM module is used in the design of GMSEMR to transmit (send) the amount of power consumed to the utility company and the consumer. It operates on a +5 -voltage power supply. The GSM module can be configured to text mode and has several inbuilt libraries which enables it to communicate with the microcontroller.

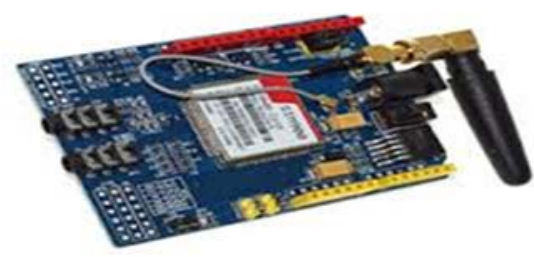

Fig. 4 GSM module

The complete circuit diagram of the design that integrates all the units of the block diagram is shown in Fig. 5 .

\section{RESULT AND ANALYSIS}

This section presents the result from the design section of this work. Following the different stages of the design, the GMSEMR prototype has been successfully implemented and tested. The GMSEMR consists of Sensors, GSM module, LCD display and varying load. An image capture of the skeletal design of the work with all the described hardware is shown in Fig. 6.

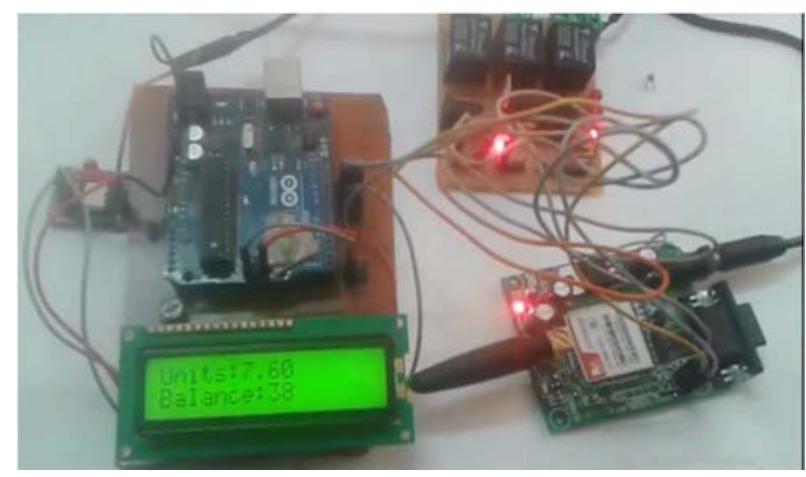

Fig. 6 Components of GMSEM

\subsection{Testing}

To test the GMSEMR system, we first test the power supply circuit by testing the rectifier, using an oscilloscope to see the waveform generated by the circuit. The waveform was found to be distorted due to ripples present in the signal. In other to remove the ripples, a filter capacitor was used to filter the wave form. The waveform generated by the power circuit before and after filtering is shown in Fig. 7 and Fig. 8 respectively

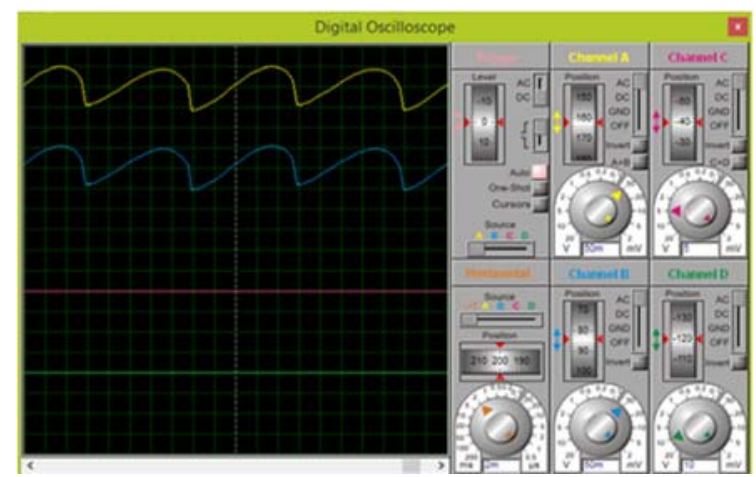

Fig. 7 Waveform by the power circuit before filtering

As shown in Fig. 7, the waveform of the signal is not a pure dc signal due to the presence of ripples, which causes noise and reduce the voltage drop across the load. However, this can be solved by introducing a filter circuit. The filter capacitor also raises the output voltage according to its rating. Fig. 8 shows the wave form generated after the introduction of a filter circuit.

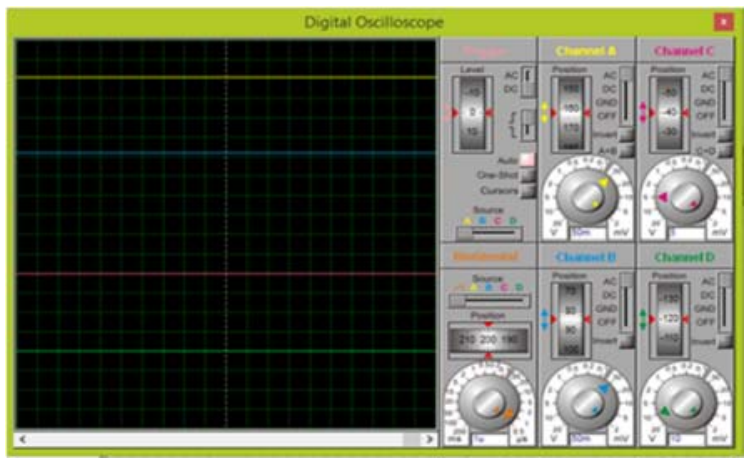

Fig. 8 Waveform by the power circuit after filtering 


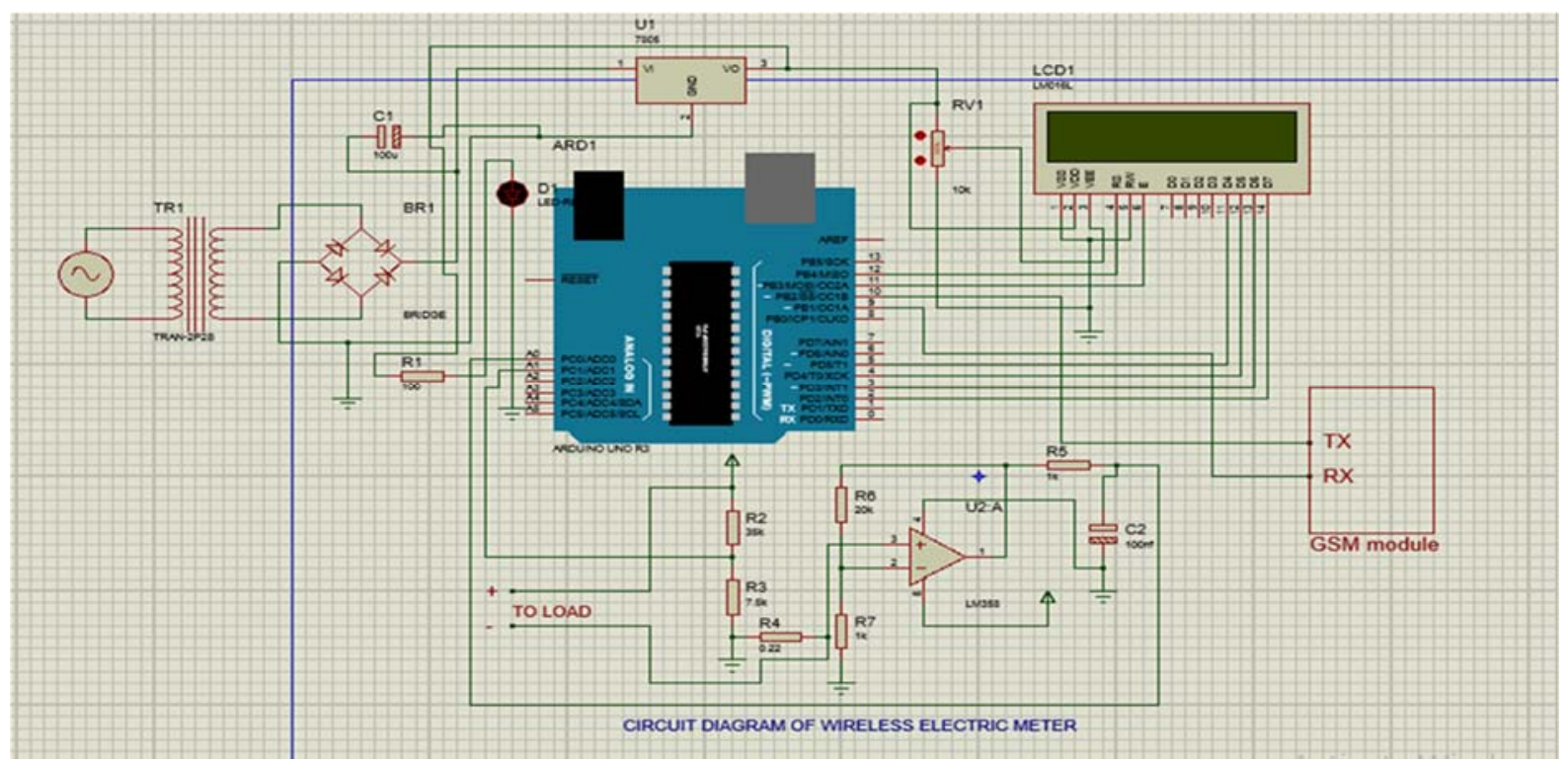

Fig. 5 Complete circuit diagram of the GMSEMR

To test the performance of GMSEMR, we power ON the system and the sensor scans for the presence or absence of a load. If no load is sensed, the liquid crystal display (LCD) displayed power $0.00 \mathrm{~W}$ on the screen, meaning the power consumed is zero. However, when a load is connected to the system through the load terminal, the power consumption of the load is displayed on the screen of the LCD. In the design, whenever the power consumption in a day is too high, the system is to alert the consumer. The reference power for a maximum consumption in a day is 2.5 watt, hence whenever the consumed power exceeds this rating, a short message is sent to the consumer and the electricity supply company. The power consumption is sent in text message format.

\subsection{Results}

The GMSEMR was connected to the source of power supply and powered. The voltage supply reading was 16.80 volt while current and power consumed were $0.00 \mathrm{amp}$ and 0.00 watt respectively. Three light bulbs connected in series were thereafter connected to the load terminal of the GMSEMR for 30 seconds. The LCD displayed 16.80 volt as the voltage reading while the current reading was 0.13 amp and power was 2.19 watt.

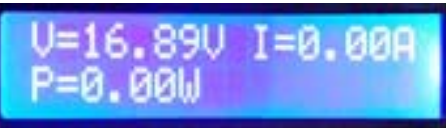

Fig. 9 A capture of the readings on LCD screen when no load was connected

Furthermore, two light bulbs connected in series were loaded and the following readings was displayed on the LCD; voltage was 16.5 volt, current $0.15 \mathrm{amp}$ and the power consumed was 2.53 watts.

Table 1 shows a summary of the readings of the smart automatic wireless electric meter
Table 1 GMSEMR Readings

\begin{tabular}{|l|c|c|c|}
\hline LOAD(S) & $\begin{array}{c}\text { VOLTAGE } \\
\text { (VOLT) }\end{array}$ & $\begin{array}{c}\text { CURRENT } \\
\text { (AMP) }\end{array}$ & $\begin{array}{c}\text { POWER } \\
\text { (WATT) }\end{array}$ \\
\hline $\begin{array}{l}\text { Without } \\
\text { load }\end{array}$ & 16.89 & 0.00 & 0.00 \\
\hline $\begin{array}{l}\text { Three light } \\
\text { bulbs } \\
\text { connected in } \\
\text { series }\end{array}$ & 16.80 & 0.13 & 2.19 \\
\hline $\begin{array}{l}\text { Two light } \\
\text { bulbs } \\
\text { connected in } \\
\text { series }\end{array}$ & 16.87 & 0.15 & 2.53 \\
\hline
\end{tabular}

The accuracy the GMSEMR was checked by comparing the readings that are displayed on the LCD of the GMSEMR and that received by SMS. The GMSEMR was also checked by connecting and disconnecting the loads. The results obtained show that the displayed reading on the LCD and SMS, sent to the billing office of the electricity provider, are the same (see Fig. 10). Furthermore, an SMS was sent to the consumer in the second experiment when the power reading was above the 2.5 watt threshold set.

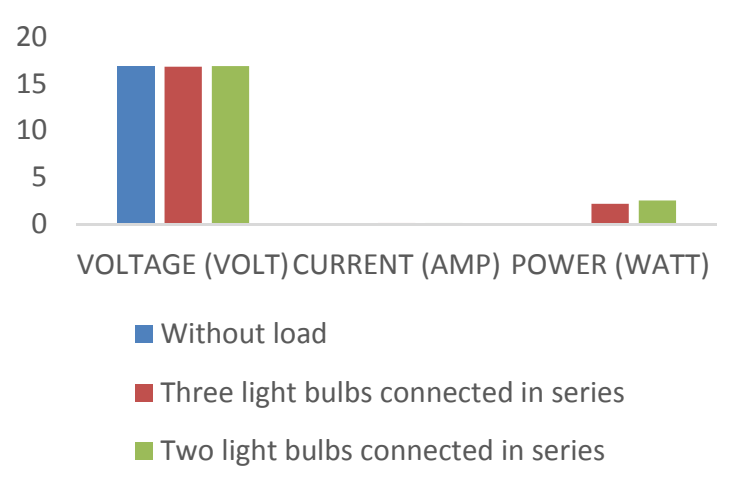

Fig. 10 Readings of the smart automatic wireless electric meter 


\subsection{Discussion}

The GMSEMR function by using a current and voltage sensor to sense the current and voltage readings. Initially, in the case of no load, the voltage at the input is displayed on the LCD screen. The current at this point is zero as a load needs to be connected in other to measure the current flowing through it. The moment a load is connected to the load terminal, the current flowing through the load is measured and displayed on the LCD screen. As power is the product of voltage and current, power depends on the voltage and the current. Power, which is the product of the voltage and current is also displayed on the LCD screen. The readings displayed on the LCD are the power consumed in watt, watt-hour and kilowatt-hour

The testing of the accuracy of the GMSEMR System was checked by comparing the readings that was displayed on the LCD of the System and that received by SMS. The GMSEMR was also checked by connecting and disconnecting the loads. This provided accurate results, hence verifying the performance and accuracy of the system.

\section{CONCLUSION}

This work has presented the design and implementation of a GSM Module-based Smart Electric Meter Reader that sends periodical reading of the amount of electricity consumed to the electricity supply company billing office and also to the consumer, wirelessly. This was achieved through software and hardware design. For the software design, Arduino IDE application software was used to write and debug programs and uploaded to the Arduino microcontroller. For the hardware design, components such as, power supply unit consisting of transformer, bridge rectifier, filter and voltage regulator; Arduino Uno (Microcontroller); Voltage Sensor; Current Sensor; Liquid Crystal Display (LCD) and GSM module were used. From the experimental result, the system accurately measures the consumed power. This was determined when the displayed bill was compared with that sent through SMS. Other notable achievements include an increase in the accuracy of the meter reading, reduction of manpower and chance of bill tempering. Furthermore, it eliminates invasion of privacy by service providers and reduces the cost of invoicing electric bill.

\section{REFERENCES}

[1] DANGE, K. M. - PATIL, S. S. - PATIL, S. P.: (2017). Prepaid energy meter using GSM module. International Journal of Engineering Science Invention, 6(2) pp. 8085.

[2] ABDULWAHAB, L.: (2009). An assessment of billing electricity consumers via analogue meters in Kano, Nigeria, by Kano electricity distribution plc. Bayero Journal of Pure and Applied Sciences, 2(1), pp. 27-33.

[3] CHARPAK, G. - GARWIN, R. L.: (2002). Megawatts and Megatons: The Future of Nuclear Power and Nuclear Weapons. University of Chicago Press.

[4] ABDULWAHAB, L.: (2009). An assessment of billing electricity consumers via analogue meters in Kano,
Nigeria, by Kano electricity distribution plc. Bayero Journal of Pure and Applied Sciences, 2(1),pp. 27-33.

[5] GARCIA, F. D. - MARAFÃO, F. P. - DE SOUZA, W. A. - DA SILVA, L. C. P.: (2017, March). Power metering: History and future trends. In 2017 Ninth Annual IEEE Green Technologies Conference (GreenTech), pp. 26-33.

[6] WERANGA, K. S. K. - KUMARAWADU, S. CHANDIMA, D. P.: (2014). Smart metering design and applications. Singapore: Springer.

[7] OSANAIYE, O. - CHEN, S. - YAN, Z. - LU, R. CHOO, K. K. R. - DLODLO, M.: (2017). From cloud to fog computing: A review and a conceptual live VM migration framework. IEEE Access, 5, pp. 8284-8300.

[8] AN, E. A. U.: (2011). Smart Meters and Smart Meter Systems: A Metering Industry Perspective.

[9] ZHENG, J. - GAO, D.W. - LIN, L.: (2013). April. Smart meters in smart grid: An overview. In 2013 IEEE Green Technologies Conference (GreenTech) (pp. 5764).

[10] BARONE, G. - BRUSCO, G. - MENNITI, D. PINNARELLI, A. - POLIZZI, G. - SORRENTINO, N. - BURGIO, A.: (2020). How Smart Metering and Smart Charging may Help a Local Energy Community in Collective Self-Consumption in Presence of Electric Vehicles. Energies, 13(16), 4163.

[11] WU, B. - TAN, N. - YU, X. P.: (2019). An Energy Metering Chip with a Flexible Computing Engine. IEEE Access, 7, pp. 46351-46362.

[12] BURUNKAYA, M. - PARS, T.: (2017, April). A smart meter design and implementation using ZigBee based wireless sensor network in smart grid. In 2017 4th International Conference on Electrical and Electronic Engineering (ICEEE) (pp. 158-162).

[13] S. R. T. MANIMALA V.: (2017). "Design and Implementation of Automatic Meter Reading Using PLC Modem," South Asian Journal of Engineering and Technology, Vol. 3, pp. 32-36.

[14] S. N. E. A. DRAGAN MLAKIĆ: (2017) "Designing Automatic Meter Reading System Using Open Source Hardware and Software," International Journal of Electrical and Computer Engineering (IJECE) Vol. 7, pp. 3282-3291.

[15] LEI, W.X. - JIANG, Y.X. - Hong, W. E. N. - XU, A. D. - ZHE, M. I. N. G. - HOU, W. J. - YIN, Y. J.: 2019. New Features of Automatic Meter Reading System: Based on Edge Computing. DEStech Transactions on Environment, Energy and Earth Sciences, (icepe).

[16] VICIANA, E. - ALCAYDE, A. - MONTOYA, F. G. - BAÑOS, R. - ARRABAL-CAMPOS, F. M. ZAPATA-SIERRA, A. - MANZANOAGUGLIARO, F.: (2018). Openzmeter: An efficient low-cost energy smart meter and power quality analyzer. Sustainability, 10(11), pp. 1-13. 
[17] MUSTAFA, M. A. - CLEEMPUT, S. - ALY, A. ABIDIN, A.: (2019). A secure and privacy-preserving protocol for smart metering operational data collection. IEEE Transactions on Smart Grid, 10(6), pp.6481-6490.

[18] KUMAR, P. - GURTOV, A. - SAIN, M. - MARTIN, A. - HA, P. H.: (2018). Lightweight authentication and key agreement for smart metering in smart energy networks. IEEE Transactions on Smart Grid, 10(4), pp.4349-4359.

Received March 17, 2020, accepted November 3, 2020

\section{BIOGRAPHIES}

Opeyemi Osanaiye received the bachelor's degree in electrical engineering from the University of Ilorin, Nigeria, in 2007, the master's degree in telecommunications engineering from the University of Sunderland, U.K., in 2011, and the Ph.D. degree in electrical engineering from the University of Cape Town, South Africa, in 2016. He was a part time Lecturer with the Cape Peninsular University of Technology, Cape Town, South Africa. He was with the University of South
Australia on a research exchange visit from 2015 to 2016 and a postdoctoral research fellow at the University of Pretoria, South Africa from 2017 to 2018. He is a Senior Lecturer with the Computer Engineering Department, Nile University of Nigeria. His research interests include, computer networks, cloud computing, wireless sensor network, fog computing, network security, voice over internet protocol technology, and cloud computing security. He is a registered COREN member and a member of IEEE.

Sunday Unogwu recently graduated from the telecommunication department, Federal University of Technology, Minna, Niger State, Nigeria. His research area includes Smart Metering, IoT and Data Communications.

Folayo A. Aina received the bachelor's degree in computer science from the University of Ilorin, Nigeria, in 2009, the master's degree in network systems from the University of Sunderland, UK., in 2011, and currently doing the Ph.D. degree in computing in Anglia Ruskin University, Chelmsford, UK. Her research interests include computer networks, wireless networks, MANET, mobile communication, and network security. 